\title{
Smart Taxi Navigation System Using IOS Application Case Study: Kuwait City
}

\author{
Abdulrahman Alkandari ${ }^{1+}$, Altaf Alshammari ${ }^{2}$ and Mishari Aljandal ${ }^{3}$ \\ ${ }^{1}$ Computer Department, College of Basic Education, (PAAET), Kuwait \\ ${ }^{2}$ Om Mabad Middle School, Ministry of Education, Kuwait \\ ${ }^{3}$ Electrical Department, High Institute of Energy, Kuwait
}

\begin{abstract}
Developers around the world always seek to contribute to make life seem easier using technology and communications. Therefore, the technology has become involved in almost all matters of the life, such as communications and transportation, where the transport is always suffering from delays and fraud, especially taxis category. This target group in the project abound always the monument between the passenger and driver operations where prices are floating. In addition, the delay caused by the large number of vehicles on the street and limited transportation paid, lead to the desire to bring this research which contributes to solving a lot of problems such as wasting time and fraud. This paper proposes an application named "TAXICAB" works on smart mobile phone that uses GPS technology to GPS and it helps to know all the information for the driver and the advantage of ease of use.
\end{abstract}

Keywords: smartphone, global position system GPS, IPhone operating system IOS.

\section{Introduction}

Information technology has become linked to the development of communities in our time, and the multiplicity of the most important means for the transfer of developing societies to more sophisticated societies. They contribute directly to building a new society based on electronic information services directly related to the production of communication and education services. Accordingly, highlights the important question centered on whether the information and communication technology offers the ability of developing countries to overcome poverty, and skip the traditional stages of development, and move it into a knowledgebased growth path and has a value of greater added. It is clear that ICT (Information and Communications Technology) alone is just a tool, and tools are not a substitute for the need for a real development. However, the information and communications technology provides tools that development and restructuring acceleration by providing a more consistent access to information, as a further people have gained access to more information whenever and wherever they need it. The effect of this is represented profound changes in the structures of markets and organizations and patterns of economic and administrative existing behavior before the Internet age. [1]

The term information technology refers to a set of elements and capabilities that are used in data and information collection, storage and dissemination using computers and communications technology at high speed and efficiency to bring something useful to help the development of societies. Information technology has changed the industrial revolution of your production system eighteenth century in terms of production volume, quality and form of the product, which can be modified based on the customer's request to keep abreast of new developments and technologies. Information technology available around us and affect and

\footnotetext{
+ Corresponding author. Tel.: +96599868868.

E-mail address: aam_alkandary@yahoo.com.
} 
improve the course of our daily lives (though not our sense of them), they are found in television stations, and institutions of transportation, office automation, financial institutions, agriculture, accounting, education and training, and at home, health, medicine, manufacturing, and the press, energy, sports, and others [2].

During the big revolution in the online world, people are trained to share their thoughts, experiences, and interests on the network. In the past five years since the launch of the Apple iPhone phone, and the dissemination of its stores around the world, grown applications mobile phones to become a huge market as a result of these electronic revolutions. Customers traveling in the Arab world these days to use the smart phone applications for faster access to services companies and entities that are dealing with. Recent studies have demonstrated the importance to be any organization, private company or government agency, or even members of the smart phone application offers the same service provided by the website of the same study, which demonstrated the growing decline in the number of users that.

\section{Problem Description}

The majority of residents are suffering difficulties in the Arab countries. Residents who do not have a private jet, face difficulties in moving from one place to another, especially because of poor public transport. Taxis are often considered in Kuwait as an easy and convenient transport and sometimes cheap. But with the presence of more than 65,000 taxi scattered across the country is likely to encounter some of the taxi drivers of others are honest and exposure to the agent fraud more than one way, do not be afraid this does not mean that everyone as well. In fact, the most honest taxi drivers are as follows months we offer you ways to fraud taxi drivers in Kuwait and how to behave in the event of any of them, As a tourist, the driver assumes that tourist do not know the right way, thus he selects the roads longer and bypass to get to the area you want and that increase the value of the fare. In case the tourist knows the short way, driver may give excuses such as the presence of a traffic jam and congestion of the usual route so he exhibits roads lightest busiest.

Also, to assume that the fare is 2 dinars and you give the driver a sheet of category 5 dinars, here the driver will demonstrate that he is looking for the remaining amount to return 4 dinars. Then he will tell you unfortunately do not own exchange or 3 dinars to restore the balance It is expected that you are a tourist or a citizen or expatriate tolerance will do the rest and thus got 3 extra dinars for free. The driver proposal fixed price fare for connection rather than using the counter, and will try to convince you that this method is cheaper and better for you because of traffic jams and congestion and it will behave shortcuts to reach as soon as possible. This command is not considered a stunt, but some taxi drivers in Kuwait illusion that they are in the Circuit precedents! Certainly they have the experience of leadership in this way where they want to gain time and to get the largest number of customers during the time, in all cases, if you feel you are in danger or that the driver recklessly

Moreover, usually represents a taxi ride crisis for any traveler with a private exploitation of some foreign drivers not knowing the price of a timely and roads shorter but with the arrival of the application, which provides taxi with determining the exact amount of the trip. Anyone can request a taxi through this free application easily using GPS technology. GPS in his personal application to be able to locate the user, and the closest taxi available. Then, it send the location for the taxi driver with high accuracy. The application is easy to call a taxi with costing; also, it is free and secure service where passengers will be able to see the image of the driver on the phone. User can follow the taxi path until it reaches the whereabouts and there is no need to tell the driver where the place depends on the location of the service as GPS.

\section{Related Works}

J. Yuan, Y. Zheng, X. Xie, and G. Sun, 2013, this paper explain that there is a way to solve all these problems, according to the rules set by the Egyptian Ministry of Interior. For the first time ever in Cairo, you can choose the driver safely from a large list of authorized drivers, and give him a proper evaluation and comment on his behavior and his way with you to help others to make the right decision [3].

In 2003, Silva, Aloizio P., and Geraldo R. Mateus in their paper present the idea of private chauffeur service on demand or "smart Taxi" provided by the company "upper" on the user to exploit the application installs on smart devices, and then register for the service by entering their personal data and credit card 
numbers, and then the user can see his geographical also appear on the map of the company "upper" and scattered cars in the neighbourhood with the estimated time of arrival. The user can then click on the screen to ask for a car, and in the case of acceptance of the application, the user can see the car as it moves towards him, and when he arrived to the destination, it can be for passengers to leave the car without paying the freight in cash, because the process is done automatically through the application that deducts the fare from the tally banking [4].

Ali, Yousif Al, Ibrahim Al Kattan, and Meftah Hrairi mention an application that selects the location of the user automatically makes getting a taxi is very easy and convenience. Once the driver confirmed to request the user is notified immediately taxi location. Upon arrival of the car to the place chosen by the user is informed of this as well.

Hla Taxi Dubai is the latest development of the transportation system in the United Arab Emirates is characterized by taxi white and grey, turquoise, where he dedicated most of the tourists through the permanent custody of the vehicle and return to an area Reservation, Reservations can be made through the application on the phone or the website or call the hotline [5].

Awajan, Albara on Sept 2013 discussed the distinguished taxi in Jordan which is the type of Mercedes Taxi system features rows of different roam the streets of the Jordanian capital Amman passenger traveling through the site to locate the nearest passenger satellite and the application on the mobile phone, this limits the application of wasting time searching for passengers[6].

M. Hadi Baaj elucidated the Taxi Beirut which is an application similar to other applications distinguishes this application through that passengers can choose its own specific driver and mobility on demand in addition to pricing Fixed night and day with free charge to the request within Beirut, but in addition, you know symbolic abroad on demand [7].

Anwar, Ayesha, Mikhail Volkov, and Daniela Rus, discussed that getting Taxi new system of practice in the State of Palestine is characterized by a system that exists in most areas of the busiest. In addition, most applications can be for the driver to know the place-bound him passenger through mapping system utter the name (Hot maps) and is characterized by taxis small size and works on the electricity system and gasoline with engines hybrid [8].

\section{Implementation}

If the user want to use taxicab services, he/she must have account by sign up. There is two type of accounts: passenger and driver. Signing up require filling some information as first name, last name, mobile number, email address, password and choose the account type of user passenger or driver as shown in Figure 1 for the DFD (Data Flow Diagram) of the application.

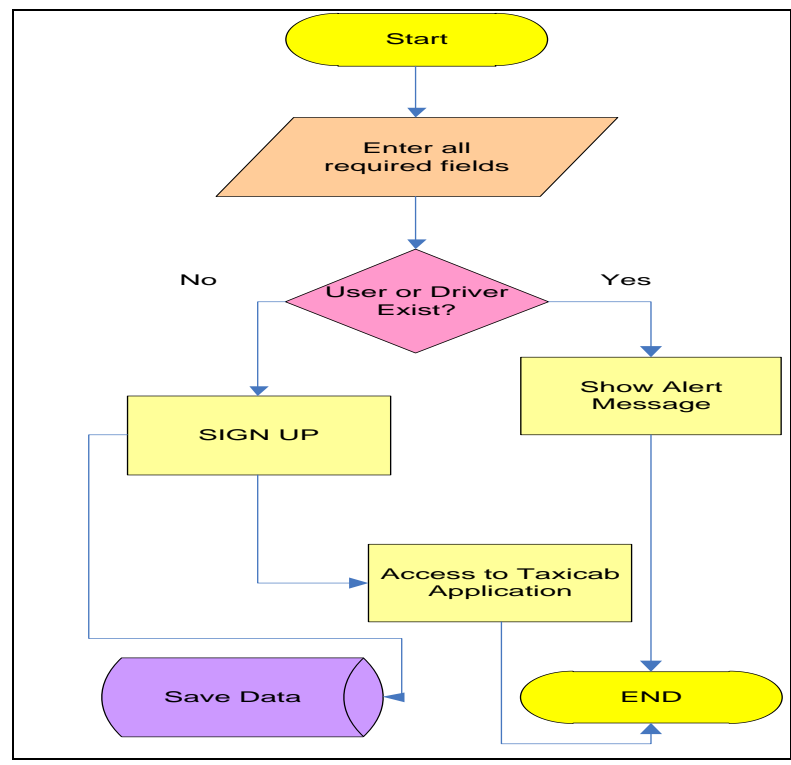

Fig. 1: Sign up for the application. 
When the user need to access to the application again, he/she must sign in by enter username and password. After that, the system check on user authentication then let the user access to the taxicab services. On the other hand, if the user forget the password the user must request the password by enter his email address. The system will check if the user exist or not then send password through email. Figure 2, explains how the application works and how it find a taxi for the user. When the user (passenger) access to the taxicab services. The system will check on location services. If the location services not enable, the system will not be able to get user location. User can enable location services in the mobile settings. On the other hand, if the location services is enable, the system will be able to find user location on the map. The second step, the system will request from user to determine his/her destination on the map then the user need to make taxi request. The system will calculate the price and the distance between user current location and destination. After that, the user will has all taxi request details. In addition, the user has two choice accept or cancel. If the user choose accept, the system will start looking for taxi. After the system complete the search process and find the nearest taxi. The system will calculate the estimated time for the driver to pick up the user then view full details about driver and ride. On the other hand, if the system cannot find the taxi on user area. The user has two choice cancel request or reload the view and try again.

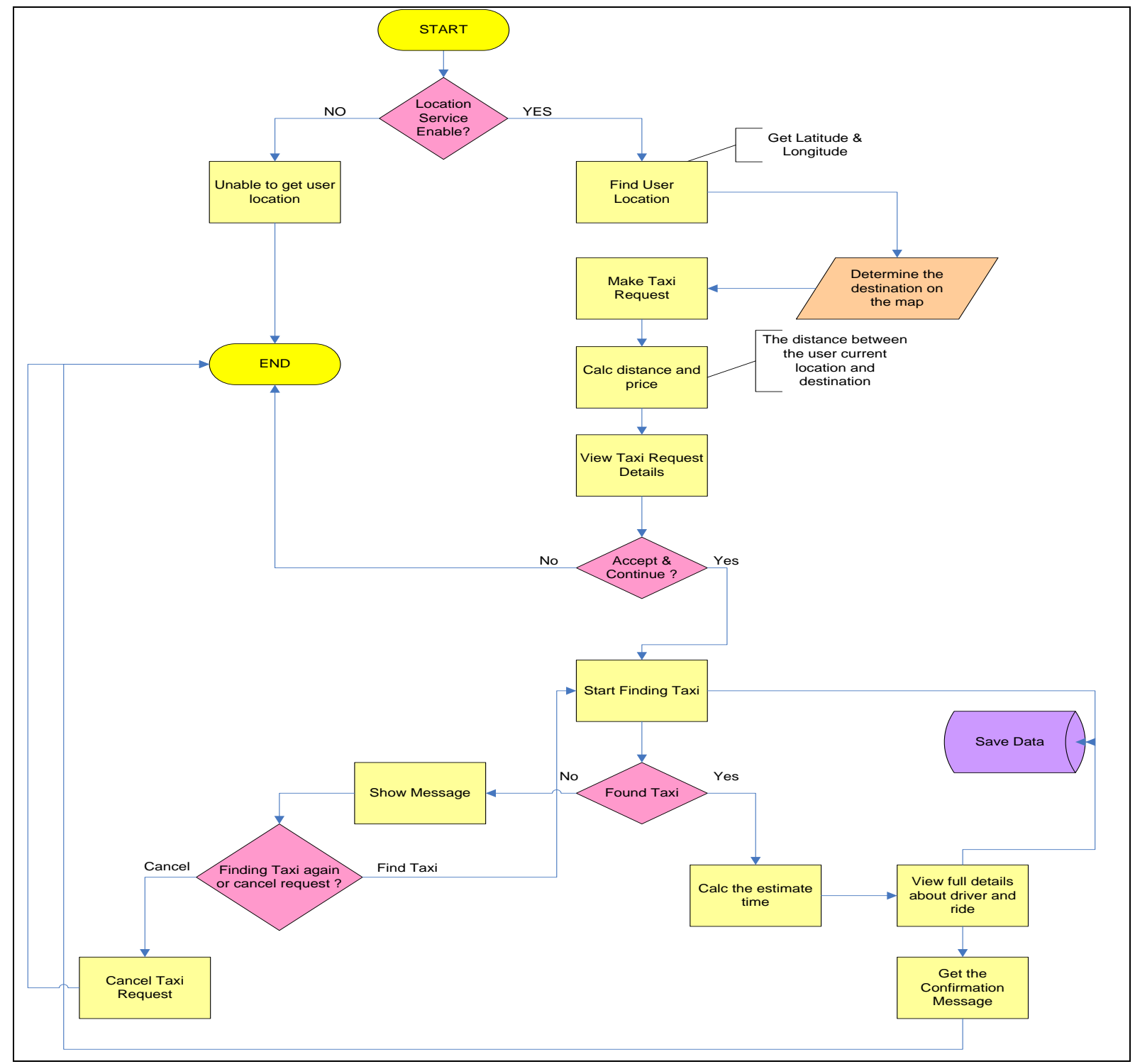

Fig. 2: Finding the taxi (passenger) DFD.

On the driver side. The system also will check on location services. If the location services is enable, the system will be able to determine driver location on the map. If the driver status is busy the system will be update the driver location on database but if the user status is available the driver will be able to get taxi 
request. If the driver get the taxi request from the system, the status will be change to busy; the red pins will be set on driver map to determine the passenger current location and destination. Finally, the driver will be able to display taxi request details. Figure 3 shows that clearly.

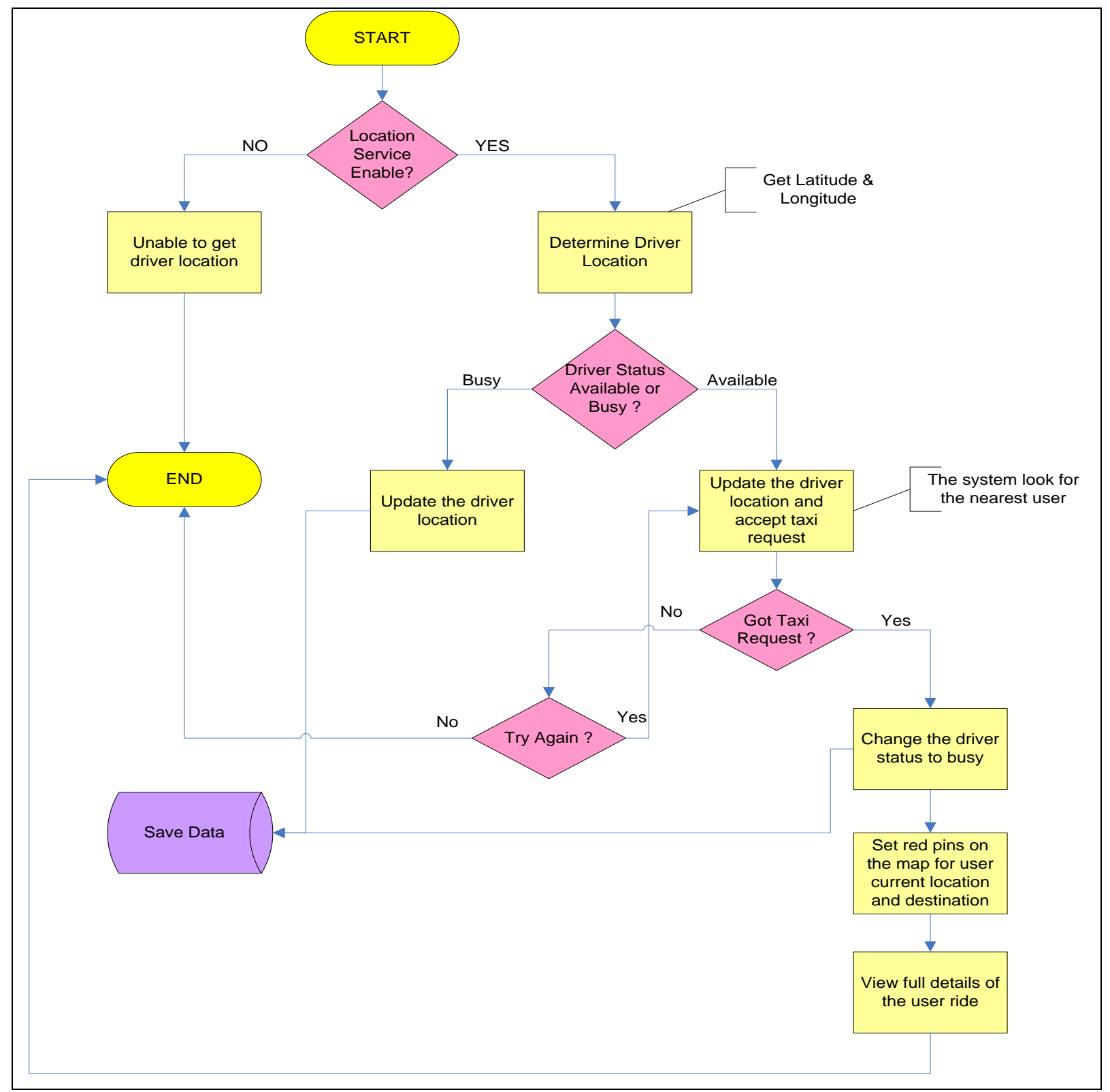

Fig. 3: Getting taxi request (Driver) DFD.

\section{TAXICAB Design}

The screen shots of the application it this paper to let the reader know the UI of the application and how its work in the mobile. In this application, the UI designed friendly to make dealing with application easier and to make the application more powerful. The user can login to taxicab by username and password after the user had been signup in the application. Additionally, the user can press the Forgot Password button for password reminder or press Sign up button in the right upside for register. To sign up, user must press Sign Up button to enter Login screen. As shown in Figure 4 the user sign up by enter the required information (first name, last name, mobile number, Email address and password). In addition, user must choose one of the application account type (User / Driver) and that by press "Account Type" which moves user to the Account Type screen. After choosing account type, user should press back button to complete sign up. Then the user should press on tick button (in sign up screen) to complete sign up process and access to the taxicab service. User can return to the Login screen by press the "back" button. 


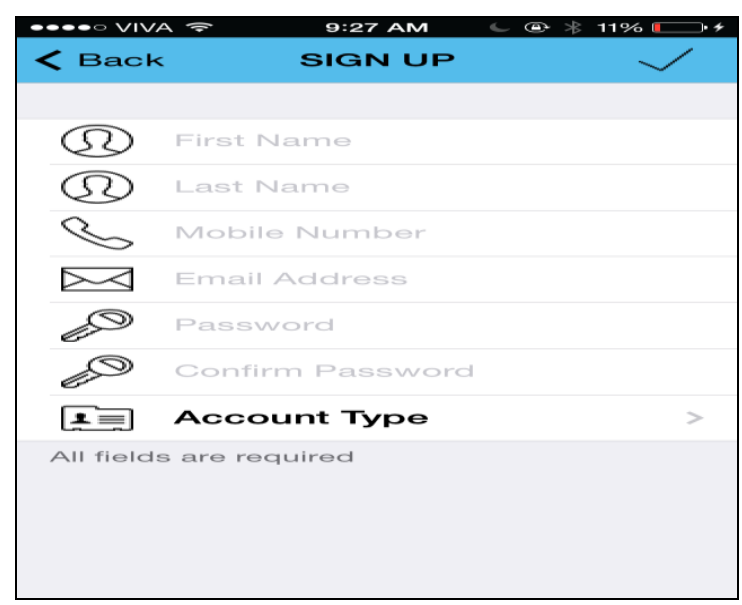

Fig. 4: Sign Up screen.

Figure 5 shows the map screen, which is showing the user current location by black dot on the map and latitude/longitude values. If the user want to make taxi request, he/she must make long press on the map to determine his/her destination then press on Make Taxi Request button. The user also can press on logout button to return to login screen.

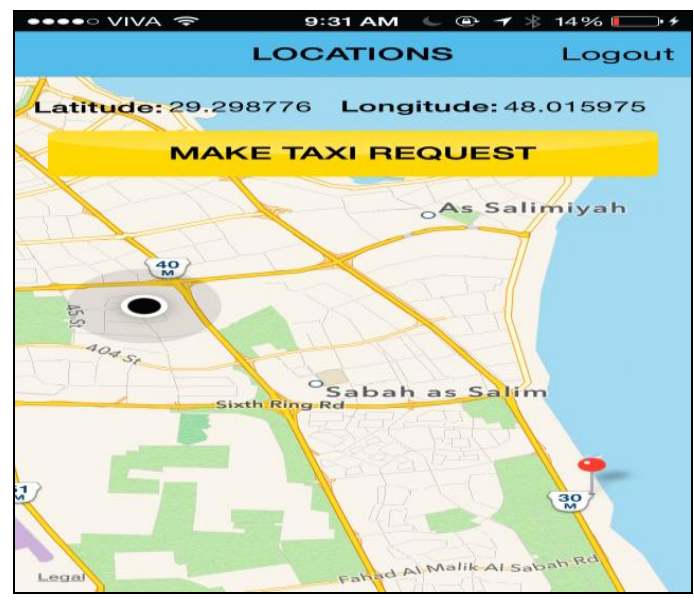

Fig. 5: User location screen.

As shown in Figure 6. After pressing on Make Taxi Request in previous screen. The system in taxicab determine country, state, user current location, user destination, distance between user current location and user destination. Therefore, the system calculate the price $($ Price $=($ Distance in $\mathrm{KM} * 0.5)+2 \mathrm{KD})$. The user for sure can accept this offer and continue on this process or press on cancel button and return to location screen.

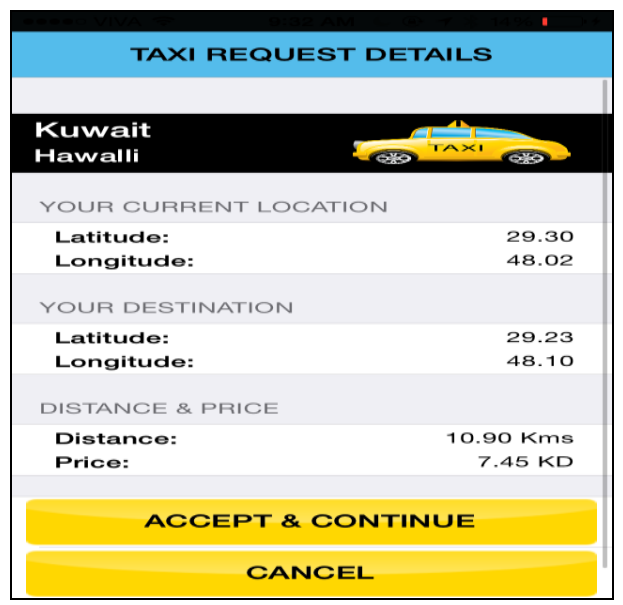

Fig. 6: Taxi request details. 
The user should wait for $20 \mathrm{sec}$ until he/she get the respond (find taxi or no taxi found). If the system cannot find taxi in 20 seconds, the user has two choice. The first choice is pressing on the "cancel request" button and return to the location screen. The second choice is pressing on the "reload" button and try again to find taxi. After complete the search process in the previous screen and find the nearest taxi. The system will display all taxi request details and calculate the estimated time for the driver to pick up the user (Estimated Time $=(($ Distance between user and driver in $\mathrm{KM} * 2)+2))$. After that, the user should press on continue button to complete the process. That shown in Figure 7.

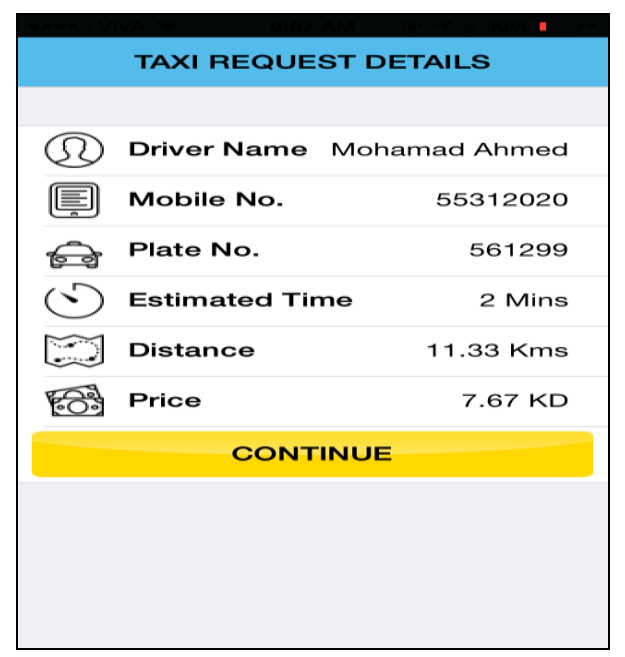

Fig. 7: Taxi request details screen.

The user get the confirmation with the estimated time for pick up. User can press on the main button to return to the location screen or press on the logout button. In Figure 8, the driver location shows the driver current location by black dot on the map and latitude/longitude values. Additionally, its shows the status of driver (Available or Busy). On this screen, the driver can press on reload button on the right upside to update his location and find taxi request. He can also press on logout or change his status (Busy if the driver cannot accept any taxi request or Available if he is ready to accept for taxi request). If the Driver status is available and someone make taxi request, the driver might be receive the notification message to let him know that there is a taxi request. Additionally, two red pins will show on the map to determine user current location and user destination. After the driver got taxi request, he can press on taxi request details button to find out the taxi request details. In the previous screen if the driver press on the "OK" button to show the taxi request details, the system will display all taxi request details (country, state, user current location, user destination, price and estimated time).

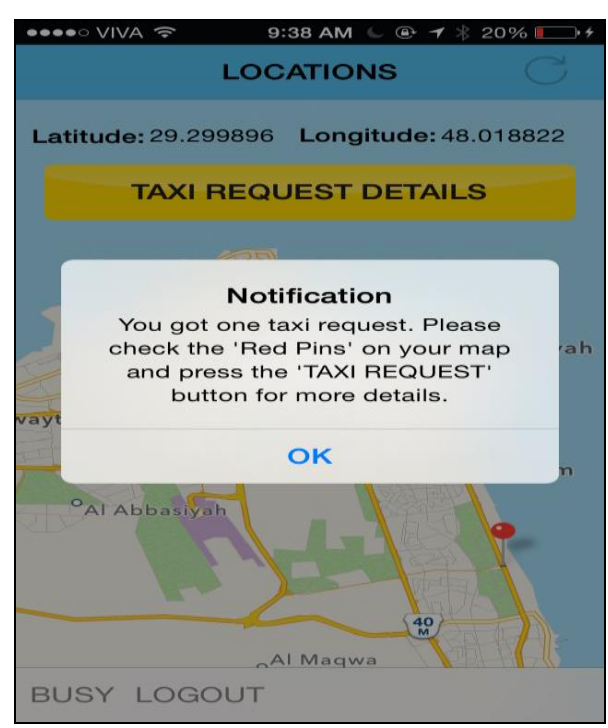

Fig. 8: Driver location screen. 


\section{Results}

After this application will select the location of the user automatically makes getting a taxi is very easy and convenience. Once the driver's confirmation of the request the user is notified immediately taxi location. Upon the arrival of the car to the place chosen by the user is informed of this as well, users will be able to allow the driver the RTA to get direct feedback from users and increase their level of satisfaction with the service performance evaluation. Offers the user a simple model of five points to evaluate the performance of the driver after the trip ends. The application serves as a channel of communication smart characterized simply infinite and keep pace with the modern techniques and serve a broader includes all segments of society and takes into account the needs and aspirations. Moreover, this smart application makes a book a taxi a new type of luxury and excellence provided for all in the State of Kuwait, that smartphone users can download the software on their phones through the Apple Store on the iPhone and phones in the Store on the Galaxy phones.

\section{Conclusion and Future Work}

The application of smart taxi is one of the most important services and the latest smart applications development and most recently will be launched. The application is the first of its kind, which gives the possibility to book taxis, tracking and arrival paths, also allows users to book a taxi in Kuwait at the touch of a button at any time and from any place where is easy to use. The new application covers all taxis in Kuwait and more than 65,000 cars travel, additionally, that the application be able to pick up the title automatically and recognize the passenger's location, which will help to identify the title, without the need for details and data may be difficult in some cases to some elderly or tourists. Also, launch the second version of the application taxi smartphone compatible with smart phones, which allows the introduction of new services to customers, including payment of traffic fines, and the introduction of credit cards in advance for discount fares, as well as it includes services in Kuwait Guide, available in Arabic and English, is available on the platform Play Store, Apple Store.

\section{References}

[1] Wegener, M. Piorkowski, M. Raya, H. Hellbruck, S. Fischer, and J.-P. Hubaux, "TraCI: An Interface for Coupling Road Traffic and Network Simulators," in 11th Communications and Networking Simulation Symposium (CNS'08), 2008 pp.841-846

[2] M. Lehtinen, A. Happonen, and J. Ikonen, "Accuracy and time to first fix using consumer-grade GPS receivers", in 16th Int. Conf. Software, Telecommunications and Computer Networks, 2008, pp.334-340

[3] Yuan, Jing, Yu Zheng, Xing Xie, and Guangzhong Sun. "T-drive: enhancing driving directions with taxi drivers' intelligence." Knowledge and Data Engineering, IEEE Transactions on 25, no. 1 (2013): 220-232.

[4] Silva, A.P. ; Mateus, G.R. Intelligent Vehicles Symposium, 2003. Proceedings. IEEE DOI: 10.1109/IVS.2003.1212877 Publication Year: 2003 , Page(s): 25 - 30 Cited by: Papers (1) | Patents (2)Anwar, A. ; Volkov, M. ; Rus, D. Intelligent Transportation Systems - (ITSC), 2013 16th International IEEE Conference on DOI: 10.1109/ITSC.2013.6728312 Publication Year: 2013 , Page(s): 694 $-701$

[5] Ali, Yousif Al, Ibrahim Al Kattan, and Meftah Hrairi. "Risk analysis factors of the emission in transportation a case study-Dubai Taxi." Engineering Systems Management and Its Applications (ICESMA), 2010 Second International Conference on. IEEE, 2010.

[6] Awajan, Albara. "An Automated Taxi Booking and Scheduling System." InModelling and Simulation (EUROSIM), 2013 8th EUROSIM Congress on, pp. 502-505. IEEE, 2013.

[7] Baaj M. 2010, "A plan for the reform and organization of the land public transport sector in Lebanon" Department of Civil and Environmental Engineering, the American University of Beirut. Beirut, Lebanon. Pp.103-113

[8] Anwar, Ayesha, Mikhail Volkov, and Daniela Rus. "ChangiNOW: A mobile application for efficient taxi allocation at airports." In Intelligent Transportation Systems-(ITSC), 2013 16th International IEEE Conference on, pp. 694-701. IEEE, 2013. 